\title{
Erratum: Multipole Love relations [Phys. Rev. D 89, 043011 (2014)]
}

\section{Kent Yagi}

(Received 22 May 2018; published 11 June 2018)

DOI: 10.1103/PhysRevD.97.129901

In Figs. 6 and 9 of this paper, we were accidentally plotting $\left|\bar{\sigma}_{2}\right| / C^{2}$ instead of $\left|\bar{\sigma}_{2}\right|$ on the vertical axis. The corrected figures are shown in Figs. 1 and 2 respectively. The fitted curves in Fig. 2 are given by Eq. (60) of this paper with the coefficients corrected to Table I.

The post-Newtonian relation between $\bar{\sigma}_{2}$ and $C$ in Eqs. (50) and (51) in this paper are corrected as

$$
\begin{aligned}
& \bar{\sigma}_{2}^{1 \mathrm{PN},(n=1)}=-\frac{1}{60}\left(1-\frac{20}{\pi^{2}}+\frac{120}{\pi^{4}}\right) \frac{1}{C^{4}}, \\
& \bar{\sigma}_{2}^{1 \mathrm{PN},(n=0)}=-\frac{1}{140} \frac{1}{C^{4}},
\end{aligned}
$$

respectively. The post-Newtonian relation between $\bar{\sigma}_{2}$ and $\bar{\lambda}_{2}$ in Eqs. (65), (70), (75) and (80) in this paper are corrected as

$$
\bar{\sigma}_{2}^{1 \mathrm{PN}}=C_{\bar{\sigma}_{2} \bar{\lambda}_{2}}\left(\bar{\lambda}_{2}^{N}\right)^{4 / 5},
$$

with

$$
\begin{aligned}
& C_{\bar{\sigma}_{2} \bar{\lambda}_{2}}^{(n=1)}=-\frac{120-20 \pi^{2}+\pi^{4}}{203^{1 / 5} \pi^{12 / 5}\left(15-\pi^{2}\right)^{4 / 5}} \approx-0.0139, \\
& C_{\bar{\sigma}_{2} \bar{\lambda}_{2}}^{(n=0)}=-\frac{1}{702^{1 / 5}} \approx-0.0124,
\end{aligned}
$$

and

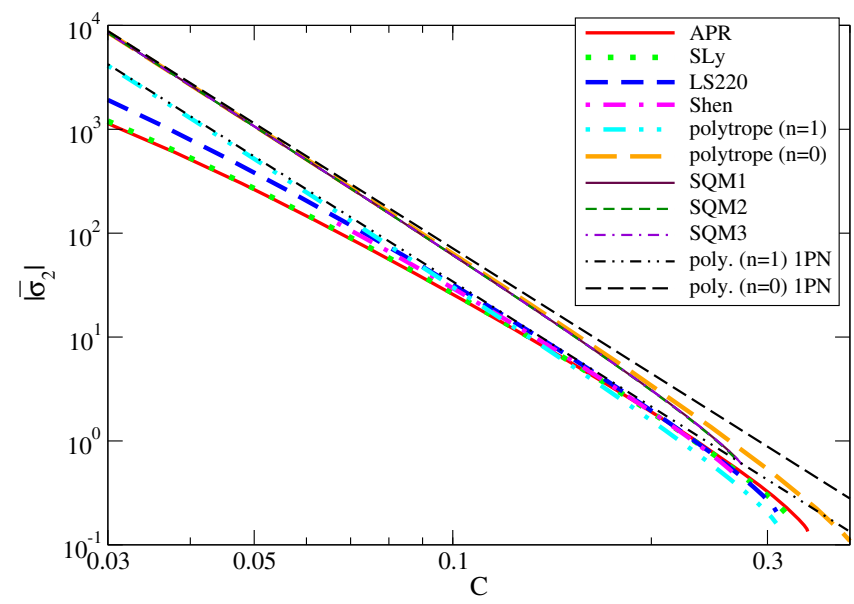

FIG. 1. $\quad \bar{\sigma}_{2}-C$ relation for neutron stars (NSs) and quark stars (QSs) with various equations of state (EoSs). We also show the relations for the $n=1$ and $n=0$ polytropes at $1 \mathrm{PN}$ order. 


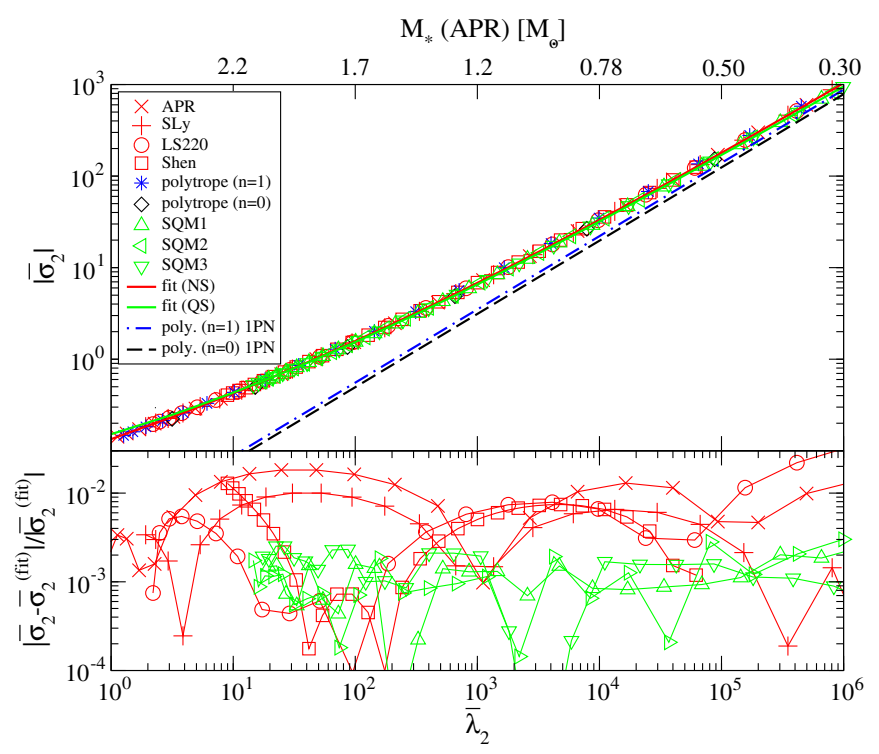

FIG. 2. $\quad \bar{\sigma}_{2}-\bar{\lambda}_{2}$ relation for NSs and QSs with various EoSs.

TABLE I. Estimated numerical coefficients for the fitting formula of the $\bar{\sigma}_{2}-\bar{\lambda}_{2}$ relation for NSs and QSs given in Eq. (61) of [1].

\begin{tabular}{|c|c|c|c|c|c|c|c|}
\hline$y_{i}$ & $x_{i}$ & NS/QS & $a_{i}$ & $b_{i}$ & $c_{i}$ & $d_{i}$ & $e_{i}$ \\
\hline \multirow[t]{2}{*}{$\left|\bar{\sigma}_{2}\right|$} & $\bar{\lambda}_{2}$ & NS & -2.01 & 0.462 & $1.68 \times 10^{-2}$ & $-1.58 \times 10^{-4}$ & $-6.03 \times 10^{-6}$ \\
\hline & & QS & -1.89 & 0.393 & $2.81 \times 10^{-2}$ & $-9.59 \times 10^{-4}$ & $1.33 \times 10^{-5}$ \\
\hline
\end{tabular}

$$
\frac{C_{\bar{\sigma}_{2} \bar{\lambda}_{2}}^{(n=0)}}{C_{\bar{\sigma}_{2} \bar{\lambda}_{2}}^{(n=1)}}=\frac{3^{1 / 5} \pi^{12 / 5}\left(30-2 \pi^{2}\right)^{4 / 5}}{7\left(120-20 \pi^{2}+\pi^{4}\right)} \approx 0.893
$$

All other results are unaffected by the above corrections.

We thank Paolo Pani for pointing out the mistakes. 